\title{
Interplay between Swampland and Bayesian Machine Learning in constraining cosmological models
}

\author{
Emilio Elizalde ${ }^{1,2, a}$, Martiros Khurshudyan ${ }^{1,2,3, b}{ }_{(\mathbb{D}}$ \\ ${ }^{1}$ Consejo Superior de Investigaciones Científicas, ICE/CSIC-IEEC, Campus UAB, Carrer de Can Magrans s/n, 08193 Bellaterra, Barcelona, \\ Spain \\ ${ }^{2}$ International Laboratory for Theoretical Cosmology, Tomsk State University of Control Systems and Radioelectronics (TUSUR), 634050 Tomsk, \\ Russia \\ ${ }^{3}$ Institute of Physics, University of Silesia, Katowice, Poland
}

Received: 2 September 2020 / Accepted: 10 April 2021 / Published online: 20 April 2021

(C) The Author(s) 2021, corrected publication 2021

\begin{abstract}
Constraints on a dark energy dominated Universe are obtained from an interplay Bayesian (Probabilistic) Machine Learning and string Swampland criteria. Unlike in previous studies, here, the field traverse itself has been used to constraint the theory and reveal its connection to the Swampland approach. The field traverse based Bayesian (Probabilistic) Learning approach is applied to two toy models. A parametrization of the Hubble constant is used for the first model, while a parametrization of the deceleration parameter is considered for the second one. The results obtained here allow to estimate how the high-redshift behavior of the Universe will affect the low-redshift one. Moreover, the adopted approach may highlight, in the future, the borders of the Swampland for the low-redshift Universe and help to develop new string-theory motivated dark energy models. The most important message from our study is a hint that the string Swampland criteria might be in tension with recent observations indicating that phantom dark energy cannot be in the Swampland. Finally, another interesting result obtained in our study is a spontaneous sign switch in the dark energy equation of state parameter when the field traverses are in the $z \in[0,5]$ redshift range, a remarkable phenomenon requiring further analysis.
\end{abstract}

\section{Introduction}

As discussed in many places, General Relativity (GR) cannot be the ultimate theory of the Universe, since it is unable to deal with situations which need to involve quantum physics (or even, maybe, an unknown theory beyond that one). The

\footnotetext{
a e-mail: elizalde@ieec.uab.es

b e-mail: khurshudyan@ice.csic.es (corresponding author)
}

problem already appeared in various viable modified theories of gravity, where quantum corrections turn out to be quite important [1-6]. One way out from this would be that General Relativity is the low-energy limit of a well-motivated (but yet unknown) high-energy UV-complete theory. In this regard, string theory may perfectly be a candidate for such UV-complete theory. It is known that it has the capacity to unify the standard model of particle physics with gravity. However, up to now the task of constructing dS vacua has hit a stonewall. Specifically, no dS vacuum could be constructed yet leading to the assumption that, in a consistent quantum theory of gravity, the dS stage does not exist [7-26]. This problem with $\mathrm{dS}$ vacua can indicate that they belong to the Swampland - the region wherein inconsistent semi-classical effective field theories inhabit ${ }^{1}$ Thus, we might have a set of consistently-looking effective quantum field theories coupled to gravity, but they could turn out to be actually inconsistent with a quantum theory of gravity. This could again indicate that dS vacua are in the Swampland [27-29].

Recently, various papers investigating the cosmological implications of the proposed Swampland criteria have appeared. ${ }^{2}$ In particular, some works have considered Swampland criteria under the form:

\footnotetext{
1 One starts from the assumption that the whole landscape of vacua provided by string theory lead to consistent effective field theories (EFT). And then, the problem with dS vacua, together with the fact that in the string landscape it is quite easy to obtain Minkowski and Anti-de Sitter solutions, leads to the conclusion that dS vacua do belong to the Swampland.

${ }^{2}$ It should be mentioned that in the recent literature there has been a lively discussion on the Swampland criteria from various perspectives, some of which can be found in [49-67], while some of relatively old discussions can be followed in the list of references in [30].
} 
1. SC1: The scalar field net excursion in reduced Planck units should satisfy the bound [27]

$$
\frac{|\Delta \phi|}{M_{P}}<\Delta \sim O(1)
$$

2. $S C 2$ : The gradient of the scalar field potential is bounded by $[28,29]$

$$
M_{P} \frac{\left|V^{\prime}\right|}{V}>c \sim O(1)
$$

or

$$
M_{P}^{2} \frac{V^{\prime \prime}}{V}<-c \sim O(1) .
$$

Provided we consider GR with the standard matter fields in the presence of a quintessence field $\phi$ to be the effective field theory. ${ }^{3}$ Here $\Delta$ and $c$ are positive constants of order one, the prime denotes derivative with respect to the scalar field $\phi$, and $M_{P}=1 / \sqrt{8 \pi G}$ is the reduced Planck mass. A study of this kind is important in order to understand when the effective field theory, admitting solutions modeling an accelerated Universe, will not end up in the Swampland. One of the most recent analysis shows that the most accurate observational data hint towards the fact that the Swampland criteria may not be valid for a dark energy dominated Universe. The result has been obtained using a Gaussian Process and recently available expansion rate data (see Table 1).

It should be mentioned that in the performed analysis, we did not use any dark energy model or a specific scalar field potential to estimate the bounds on the Swampland criteria. This owes to the fact that, applying a Gaussian Process, it is indeed possible to reconstruct the expansion rate and its highorder derivatives directly from the data, and then use them into the subsequent estimation process. On the other hand, it should be mentioned that the combination of reconstruction and estimation shows also that we could well start from the Swampland and end up in the Swampland. But, under some conditions, we might start from the Swampland and do not end up in it, eventually. And also the other possibilities exist: to start not in the Swampland and end up either inside or still outside of it [30]. ${ }^{4}$

Moreover, we would like to mention that our future analysis involving $Q=3 b H \rho_{d m}$ and $Q=3 b H \rho_{d e}$ linear non-gravitational interactions did not indicate any significant change from our previous study presented in [30] to be reported. Therefore, we suppressed and did not continue

\footnotetext{
3 The Swampland criteria above demand that the field traverses a large distance, in order to have the domain of validity of the effective field theory and $C S 2$ to be fulfilled.

4 This comes from the accepted interpretation of the results from the Gaussian process.
}

research in this direction. However, it should be mentioned that in general the non-gravitational interaction can efficiently solve various problems. One of the problems is the $H_{0}$ tension problem intensively discussed in the recent literature. ${ }^{5}$ In the light of the provided discussion it is interesting to study the cases when we have sign-changing and non-linear interactions, to understand eventually where the EFT can reside and how the initial and final locations can be changed. Of course, this is a very interesting study to be done, but we leave it as a subject of our next paper. Alternatively, here we would like to discuss how the Bayesian (Probabilistic) Machine Learning can turn the field traverse into a powerful tool allowing to constrain not only the theory but also find answers to various problems, which following standard approaches would be very costly. They still would be costly if we were to apply an ordinary Gaussian Process, as it was done in [30].

Before continuing our discussion, we would like to clearly stress that there is a huge difference between the two approaches used to address the same problem and each of them has its value. However, the way we choose to incorporate the Swampland criteria and the Bayesian (Probabilistic) Machine Learning by its nature is unique and allows to break up the limits arising in usual Gaussian Processes. The previous considerations are an indication that an extra effort is still required to understand the way how inconsistent theories should be separated from the consistent ones. Moreover, thanks to the ongoing analysis of the most recent observational data, and most probably with the new higher-redshift data, the situation will change and new models of the dark energy dominated Universe will be developed. Brand new interpretations for the dark energy will probably appear too. ${ }^{6}$

The topic is important and interesting, but still contains various dark sides to be studied. The specific goal of this paper is to show how Bayesian (Probabilistic) Machine Learning can turn the field traverse into a very useful knowledge, what cannot be done by means of standard tools. In particular, we will establish a framework allowing to constrain cosmological models by using the string Swampland criteria, Eqs. (1) and (2) (or Eq. (3)). Probably, a study of this kind could not have been performed some time ago, but the situation now is different, as we shall see. Moreover, it should be mentioned that it is still not possible to do the same by means of Bayesian analysis, where the likelihood function has to be evaluated. What renders the analysis here now

\footnotetext{
$\overline{5}$ We mentioned only the $H_{0}$ problem since in this paper we discuss it taking into account the interplay between the Bayesian (Probabilistic) Machine Learning and Swampland criteria. On the other hand, some discussion on how the $H_{0}$ problem can be solved may be found in [3145].

${ }^{6}$ For this moment this can be performed with the Bayesian (Probabilistic) Machine Learning, which is one of the most powerful forecasting tools developed so far.
} 
Table $1 H(z)$ and its uncertainty $\sigma_{H}$, in units of $\mathrm{km} \mathrm{s}^{-1} \mathrm{Mpc}^{-1}$. The upper panel corresponds to 30 samples deduced from the differential age method. The lower panel, to 10 samples obtained from the radial
BAO method. The table is in accordance with [68] (see also references therein for more details)

\begin{tabular}{|c|c|c|c|c|c|}
\hline$z$ & $H(z)$ & $\sigma_{H}$ & $z$ & $H(z)$ & $\sigma_{H}$ \\
\hline 0.070 & 69 & 19.6 & 0.4783 & 80.9 & 9 \\
\hline 0.090 & 69 & 12 & 0.480 & 97 & 62 \\
\hline 0.120 & 68.6 & 26.2 & 0.593 & 104 & 13 \\
\hline 0.170 & 83 & 8 & 0.680 & 92 & 8 \\
\hline 0.179 & 75 & 4 & 0.781 & 105 & 12 \\
\hline 0.199 & 75 & 5 & 0.875 & 125 & 17 \\
\hline 0.200 & 72.9 & 29.6 & 0.880 & 90 & 40 \\
\hline 0.270 & 77 & 14 & 0.900 & 117 & 23 \\
\hline 0.280 & 88.8 & 36.6 & 1.037 & 154 & 20 \\
\hline 0.352 & 83 & 14 & 1.300 & 168 & 17 \\
\hline 0.3802 & 83 & 13.5 & 1.363 & 160 & 33.6 \\
\hline 0.400 & 95 & 17 & 1.4307 & 177 & 18 \\
\hline 0.4004 & 77 & 10.2 & 1.530 & 140 & 14 \\
\hline 0.4247 & 87.1 & 11.1 & 1.750 & 202 & 40 \\
\hline 0.44497 & 92.8 & 12.9 & 1.965 & 186.5 & 50.4 \\
\hline 0.24 & 79.69 & 2.65 & 0.60 & 87.9 & 6.1 \\
\hline 0.35 & 84.4 & 7 & 0.73 & 97.3 & 7.0 \\
\hline 0.43 & 86.45 & 3.68 & 2.30 & 224 & 8 \\
\hline 0.44 & 82.6 & 7.8 & 2.34 & 222 & 7 \\
\hline 0.57 & 92.4 & 4.5 & 2.36 & 226 & 8 \\
\hline
\end{tabular}

possible is the significant development in machine learning [46-48] (and references therein) occurred recently. In particular, when we consider the Bayesian (Probabilistic) Machine Learning approach that uses a model-based generative process, it becomes possible to perform the task of constraining the cosmological model by involving the string Swampland criteria presented above. Still, the constraints will need to be validated, in order to claim that the method works. In this regard, we can use one of the available observational datasets, in the way it will be shown in this work. Which could be namely a starting point to reconstruct new relevant dark energy models, inspired by or directly coming from string theory. This could also provide a reliable starting point to resolve the possible tension between the string Swampland criteria and the available observational data.

More specifically, in this paper we will use Bayesian (Probabilistic) Machine Learning and string Swampland criteria for constraining and forecasting purposes. We consider two simple toy models involving parametrizations of the Hubble and the deceleration parameters, respectively. Moreover, in both cases, the results of the fit from the Swampland criteria are compared with the ones obtained from the generated expansion rate based analysis. The results of the fit are then validated using the best expansion rate data available. Moreover, in our study we are not limited by the specific char- acteristics and details of the observations since a generative process has been used. Even then, we are able to improve the theoretical models involving information incorporated into the higher-redshift evolution of the Universe. This can also be used for forecasting purposes allowing to improve and optimize the design of the observational missions. Having taken all this into account, we have been led to consider the following two redshift ranges: $z \in[0,2.5]$ covering the available $H(z)$ expansion rate data, and $z \in[0,5]$, an extended redshift range to cover future missions.

We will report all the results obtained in order to properly stress the strongest and also the weakest aspects of our approach. One should note that our conclusions will be based on two toy models, only. But the procedure can be extended, revealing the full power of the approach. For the moment, the main message of our study indicates that GR with standard matter fields and in the presence of a quintessence field, $\phi$, is most likely in tension with the recent form of the string Swampland criteria, Eqs. (1) and (2) (or Eq. (3)). We should note that, most likely, this situation will not change significantly with higher-redshift data. Moreover, we have got a reasonable confirmation that our approach here can indeed serve as a tool for deciding if the model is in the Swampland or not. 
The paper is organized as follows. In Sect. 2 we introduce the models and obtain the equations that explicitly show how the generative process based on each model is constructed. On the way, we briefly discuss the most crucial aspects of the Bayesian (Probabilistic) Machine Learning approach, showing why it is an attractive method and how it can be used to constrain cosmological models by involving string Swampland criteria. More specifically how the field traverse can be turned into a tool to achieve the wanted results. We should remark that all this is presented in a very compact form, addressing only some crucial aspect of the philosophy behind the method and addressing the readers to available references for more details. In Sect. 3 we discuss the results obtained for the two redshift ranges $z \in[0,2.5]$ and $z \in[0,5]$. The first covers recently available $H(z)$ data for the expansion rate, which are useful to validate the results of our fit. The other redshift range is designed to cover the results to be obtained in future observational projects. Finally, in Sect. 4 we summarize the results of our analysis, followed by a discussion and final conclusions.

\section{The model and method}

In this section we will introduce the models and the method we have used in our analysis. Our starting point is GR with standard matter fields in the presence of a quintessence field, $\phi$, considered to be the EFT, in the philosophy explained in the previous section. It is known that, in this case, the EFT will be described by the following action

$$
S=\int d^{4} x \sqrt{-g}\left(\frac{M_{P}^{2}}{2} R-\frac{1}{2} \partial_{\mu} \phi \partial^{\mu} \phi-V(\phi)\right)+S_{m},
$$

where $S_{m}$ corresponds to standard matter, $M_{P}=1 / \sqrt{8 \pi G}$ is the reduced Planck mass, $R$ the Ricci scalar, $\phi$ the field, and $V(\phi)$ the field potential. On the other hand, when we consider the FLRW universe, the dynamics of the scalar field's dark energy and dark matter will be described by the equations

$$
\begin{aligned}
& \dot{\rho}_{\phi}+3 H\left(\rho_{\phi}+P_{\phi}\right)=0, \\
& \dot{\rho}_{d m}+3 H \rho_{d m}=0 .
\end{aligned}
$$

Moreover, Eqs. (5) and (6) are the energy conservation laws for the components describing the background dynamics. ${ }^{7}$ Furthermore, $\rho_{\phi}, \rho_{d m}$ and $P=P_{\phi}$ are related to each other

\footnotetext{
7 The form of Eqs. (5) and (6) shows the absence of a coupling between the scalar field's dark energy and dark matter. In general, a coupling modifying the right hand side of Eqs. (5) and (6) is present, which corresponds to an interaction between dark energy and dark matter.
}

through the Friedmann equations, as follows

$H^{2}=\frac{1}{3}\left(\rho_{\phi}+\rho_{d m}\right)$,

and

$\dot{H}+H^{2}=-\frac{1}{6}\left(\rho_{\phi}+\rho_{d m}+3 P_{\phi}\right)$.

Now, when we assume that the scalar field is spatially homogeneous, for its energy density and pressure, we have

$\rho_{\phi}=\frac{1}{2} \dot{\phi}+V(\phi)$

and

$P_{\phi}=\frac{1}{2} \dot{\phi}-V(\phi)$

where the dot means derivative w.r.t to the cosmic time, while $V(\phi)$ is the potential of the scalar field. In all equations above, $H=\dot{a} / a$ is the Hubble parameter. On the other hand, it is easy to see, from Eqs. (9) and (10), that

$\dot{\phi}^{2}=\rho_{\phi}+P_{\phi}$

while

$V(\phi)=\frac{\rho_{\phi}-P_{\phi}}{2}$.

Now, as from Eq. (6) we have $\rho_{d m}=3 H_{0}^{2} \Omega_{0}(1+z)^{3}$, then from Eq. (7) we can determine the energy density of the scalar field, which in this case reads

$\rho_{\phi}=3 H^{2}-3 H_{0}^{2} \Omega_{0}(1+z)^{3}$,

where $H_{0}$ is the Hubble parameter value at $z=0$ ( $z$ being the redshift). After some algebra, we get that $P_{\phi}=2(1+$ z) $H H^{\prime}-3 H^{2}$, where the prime denotes derivative wrt the redshift. On the other hand, we see immediately that $\rho_{\phi}^{\prime}=$ $6 H H^{\prime}-9 H_{0}^{2} \Omega_{0}(1+z)^{2}$ and $P_{\phi}^{\prime}=2(1+z)\left(H^{\prime 2}+H H^{\prime \prime}\right)-$ $4 H H^{\prime}$. Coming back to the form of $S C 1$ and $S C 2$ to be used in the model based generative process, we need only take into account that $d V(\phi) / d \phi=(d V / d z) /(d \phi / d z)$, where $d \phi / d z$ is calculated from Eq. (11), and that $\dot{\phi}=-(1+$ z) $H \phi^{\prime}$. This was the starting point of [30], where we used the reconstructed $H, H^{\prime}$ and $H^{\prime \prime}$ to do model-independent analysis and estimations.

In this paper we work with the same equations as discussed above, but use instead new parameterizations for $H(z)$. In particular, we consider two toy models. The first model uses a parameterization for the Hubble rate, while in the second case we use one for the deceleration parameter. As we already have 
at hand the last element required to organize the generative process, let us now pause a bit, in order to understand how the Bayesian (Probabilistic) Machine Learning works and how exactly is used in our analysis. In particular, we proceed through the following three steps:

- We define the model, to be used to provide a so-called generative process. In our case it will be the string Swampland criteria.

- We envisage the data to be the data obtained from the generative process. $^{8}$

- We run the learning algorithm, to get a brand new distribution over the model parameters and update our prior believe.

It should be mentioned that, within the adopted approach, one can break some of the usual limits appearing in previous methods. On the other hand, this provides a good opportunity to perform more complex studies in cosmology and astrophysics, and also in other research fields. We have already mentioned above that we just give here a very short description of the philosophy and do not go into deeper details related with the way how one can construct a model-based generative process. The process by itself is already of much interest for programmers and for computer science and data science researchers; but for us it is more important to concentrate all our attention on the physics behind the problem. After some tests, we eventually found that it is best to use PyMC3 probabilistic programming, a python-based framework, which in practice has proven to be very fast, useful, and able to be easily integrated with other python-based frameworks [69]. It uses Theano, ${ }^{9}$ that is, a deep learning python-based library, to construct probability distributions and implement cutting edge inference algorithms. We do have an additional reason to omit specific discussions on the mathematical framework behind deep learning algorithms and Bayesian Machine Learning: the PyMC3 standard tutorials are endowed with numerous excellent examples demonstrating how the above ideas can actually be implemented within a given practical problem.

It is well known that Bayesian Machine Learning is a tool allowing to reason about probabilities using probabilities. It means that it takes into account all possible uncertainties during generating and learning process. In other words,

\footnotetext{
${ }^{8}$ We address the readers to $[47,48]$ for more details about the difference between Machine Learning and Bayesian Machine Learning that rose namely in this step. We used 10 chains and in each chain generated/simulated 10000 "datasets" to cover $z \in[0,2.5]$ and $z \in[0,5]$ redshift ranges. The "datasets" have been generated using Eq. (1) taking into account its connection with $\rho_{\phi}$ and $P_{\phi}$ given by Eq. (11) and that $\dot{\phi}=-(1+z) H \phi^{\prime}$. As it is mentioned in Sect. 2, Eq. (1) eventually it would be expressed in terms of $H(z), H^{\prime}(z)$ and $H^{\prime \prime}(z)$.

${ }^{9} \mathrm{http}: / /$ deeplearning.net/software/theano.
}

it turns any bias/uncertainty into a probability improving learned results. This is one of the first advantage over other approaches used to constrain the cosmological models. However, it is an important issue to understand up to what extend this is useful. In this regard Explainability Machine Learning can be useful allowing to explain and understand obtained results. Another most interesting aspect making Bayesian Machine Learning very attractive is the learning of the constraints without evaluating the likelihood. This makes it to be a unique tool for forecasting purposes and speeds up the learning process.

\section{Numerical results and analysis}

In this section we will discuss the results. For convenience, we have divided it into two sections. Before starting with the presentation and discussion of our results we should mention that our initial study shows that both string Swampland criteria, namely $S C 1$, Eq. (1), and $S C 2$, Eq. (2) (or Eq. (3)), can be used in our generative process. Moreover, in both cases we get well consistent results. However, it is reasonable to concentrate on the generative process based on $C S 1$, Eq. (1), the reason being that the generative process is computationally less costly in this case than if we use $S C 2$, Eq. (2) (or Eq. (3)). This can be also seen from the structure of the problem and the number of mathematical expressions to be evaluated in order to use $S C 2$.

\subsection{Model with a given $H(z)$ parameterization}

The first toy model constrained in this work, using the string Swampland criterion $S C 1$, Eq. (1), is described by the following expansion rate

$H(z)=H_{0}+H_{1} \frac{z^{2}}{1+z}$,

where $H_{0}$ and $H_{1}$ are two parameters to be determined, while $z$ is the redshift. This specific parameterization of the Hubble parameter has been found from more general parameterizations using the Bayesian (Probabilistic) Machine Learning approach. Once the form of $H(z)$ is known, we can calculate higher-order derivatives and use them to start the analysis of the model by employing the $S C 1$ criterion (owing to the reasons mentioned at the beginning of this section).

The contour maps of the model given by Eq. (14), for $z \in[0,2.5]$ and $z \in[0,5]$, respectively, can be found in Fig. 1. We observe that:

- When we consider $z \in[0,2.5]$, then the best fit values and $1 \sigma$ errors are $\Omega_{d m}=0.322 \pm 0.01, H_{0}=72.59 \pm$ $1.21 \mathrm{~km} / \mathrm{s} / \mathrm{Mpc}$ and $H_{1}=90.34 \pm 1.61 \mathrm{~km} / \mathrm{s} / \mathrm{Mpc}$. 


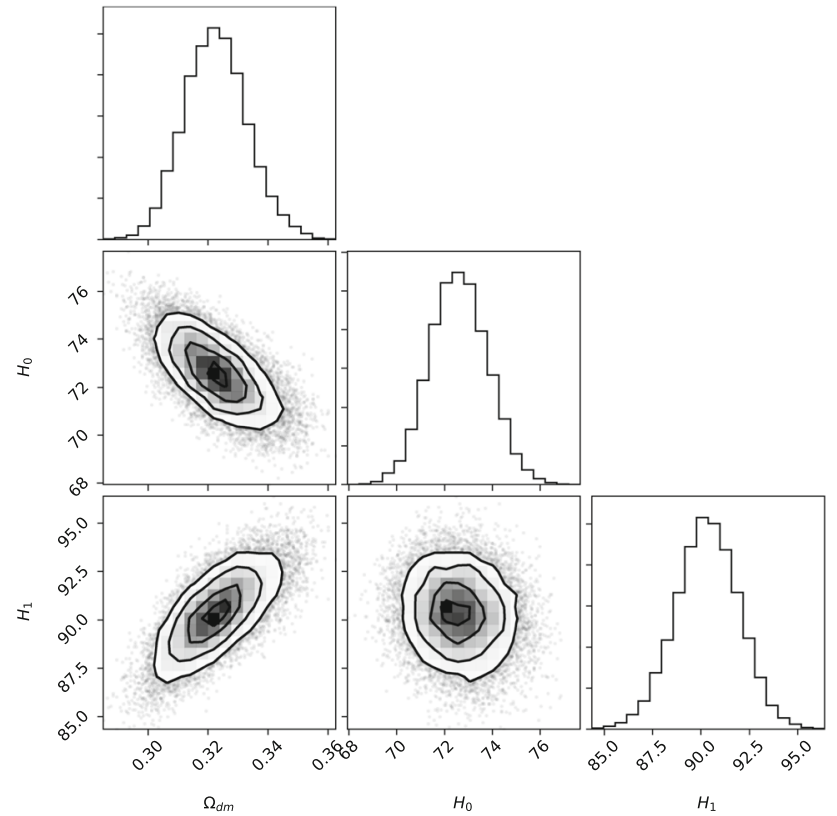

Fig. 1 The contour map of the model given by Eq. (14), for $z \in[0,2.5]$, is given on the left hand side plot. The one on the right hand side depicts the contour map of the same model for $z \in[0,5]$. The best fit values of the model parameters are $\Omega_{d m}=0.322 \pm 0.01$, $H_{0}=72.59 \pm 1.21 \mathrm{~km} / \mathrm{s} / \mathrm{Mpc}$ and $H_{1}=90.34 \pm 1.61 \mathrm{~km} / \mathrm{s} / \mathrm{Mpc}$ for $z \in[0,2.5]$. For $z \in[0,5]$, they are found to be $\Omega_{d m}=0.325 \pm 0.012$,

- When we consider $z \in[0,5]$, then the best fit values and $1 \sigma$ errors are $\Omega_{d m}=0.325 \pm 0.012, H_{0}=72.55 \pm 1.06$ $\mathrm{km} / \mathrm{s} / \mathrm{Mpc}$ and $H_{1}=89.43 \pm 1.8 \mathrm{~km} / \mathrm{s} / \mathrm{Mpc}$.

From these results, it is clear that Bayesian (Probabilistic) Machine Learning using $S C 1$, Eq. (1), certainly imposes very tight constraints on $\Omega_{d m}$ at $z=0$. Moreover, we observe that, most likely, higher redshift related field traverse will not affect the constraints on $\Omega_{d m}$ at $z=0$. Another interesting result that immediately captures our attention is the constraint obtained for $H_{0}$, which according to the parametrization considered, corresponds to the Hubble parameter at $z=0$. In particular, we found that the $H_{0}$ tension can be solved within $1 \sigma$. In other words, the model can be useful to alleviate the $H_{0}$ tension problem efficiently. ${ }^{10}$ We see also that, most likely, higher-redshift related field traverse will affect the constraints on $H_{0}$ and $H_{1}$.

From our analysis, we can extract some additional messages. First, we see that the model considered does in fact explain the accelerated expansion of our Universe, and clearly indicates the transition to this evolutionary phase, as well. Moreover, using the best fit values of the parameters of the model, the top-right plot of Fig. 2 shows that the model

\footnotetext{
10 We refer to [46-48] for more details about what the Bayesian (Probabilistic) Machine Learning says about the $H_{0}$ tension problem.
}

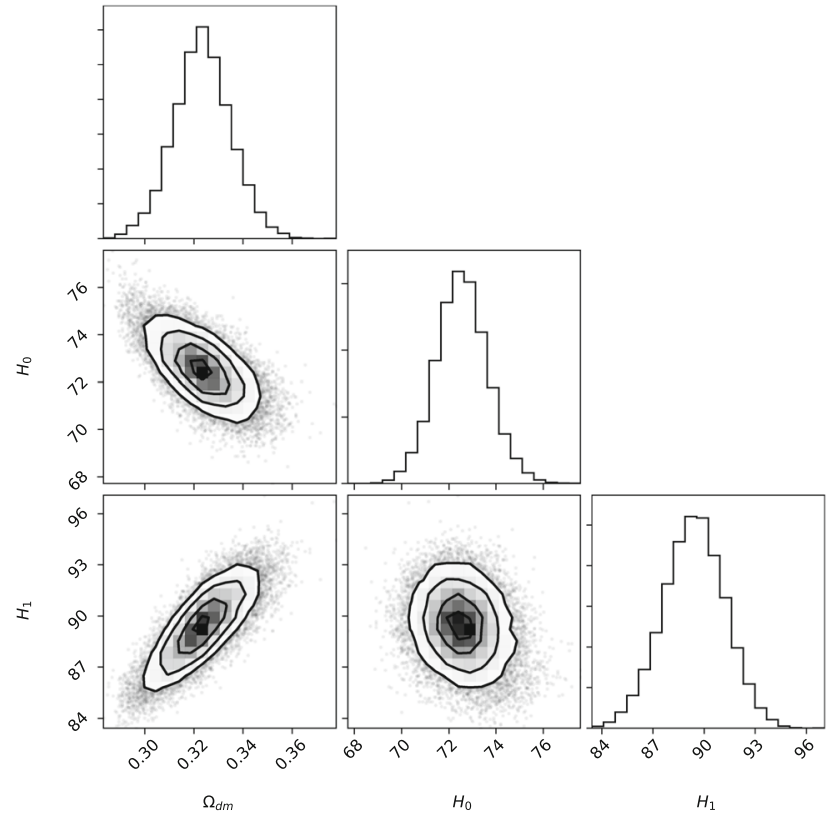

$H_{0}=72.55 \pm 1.06 \mathrm{~km} / \mathrm{s} / \mathrm{Mpc}$ and $H_{1}=89.43 \pm 1.8 \mathrm{~km} / \mathrm{s} / \mathrm{Mpc}$. The generative process has been constructed using SC1, Eq. (1), where $H(z)$ given by Eq. (14) has been used to get $H^{\prime}$ and $H^{\prime \prime}$. We refer to the PyMC3 documentation for more details on how the marginalization and the error estimations can be achieved during the learning process

can also explain the BOSS result for the Hubble parameter, at $z=2.34$.

Now, before presenting the second part of our observations, we would like to highlight once more that, starting from $S C 1$, Eq. (1), we have constrained the background dynamics of the model described by Eq. (4) and replaced the scalar field by a convenient parameterization of the Hubble constant. This allows to estimate the behavior of the $\omega_{\phi}=P_{\phi} / \rho_{\phi}$ and $S C 2$, Eq. (2), too. The results can be found on the bottom panel of Fig. 2 indicating that, at low redshift, a dark energy dominated Universe will not end up in the Swampland. However, the behavior of $\omega_{\phi}=P_{\phi} / \rho_{\phi}$ gives hints on the tension that may arise between string Swampland criteria and GR with standard matter and quintessence dark energy. Since we found that, according to the best fit values of the model parameters, dark energy has a phantom nature. Moreover, another interesting massage arises from our study, this time indicating that it is possible to avoid being in the Swampland; although in this case dark energy should have a phantom nature. In a way, this should be not surprising. Moreover, a hint about a possible tension between Swampland criteria and $H(z)$ data can be guessed from the second part of Table 2, where the constraints on the $H_{0}$ and $H_{1}$ parameters have been obtained using a generative process based on Eq. (14).

To end this section, let us summarize where from we started and what we have accomplished at this point. 

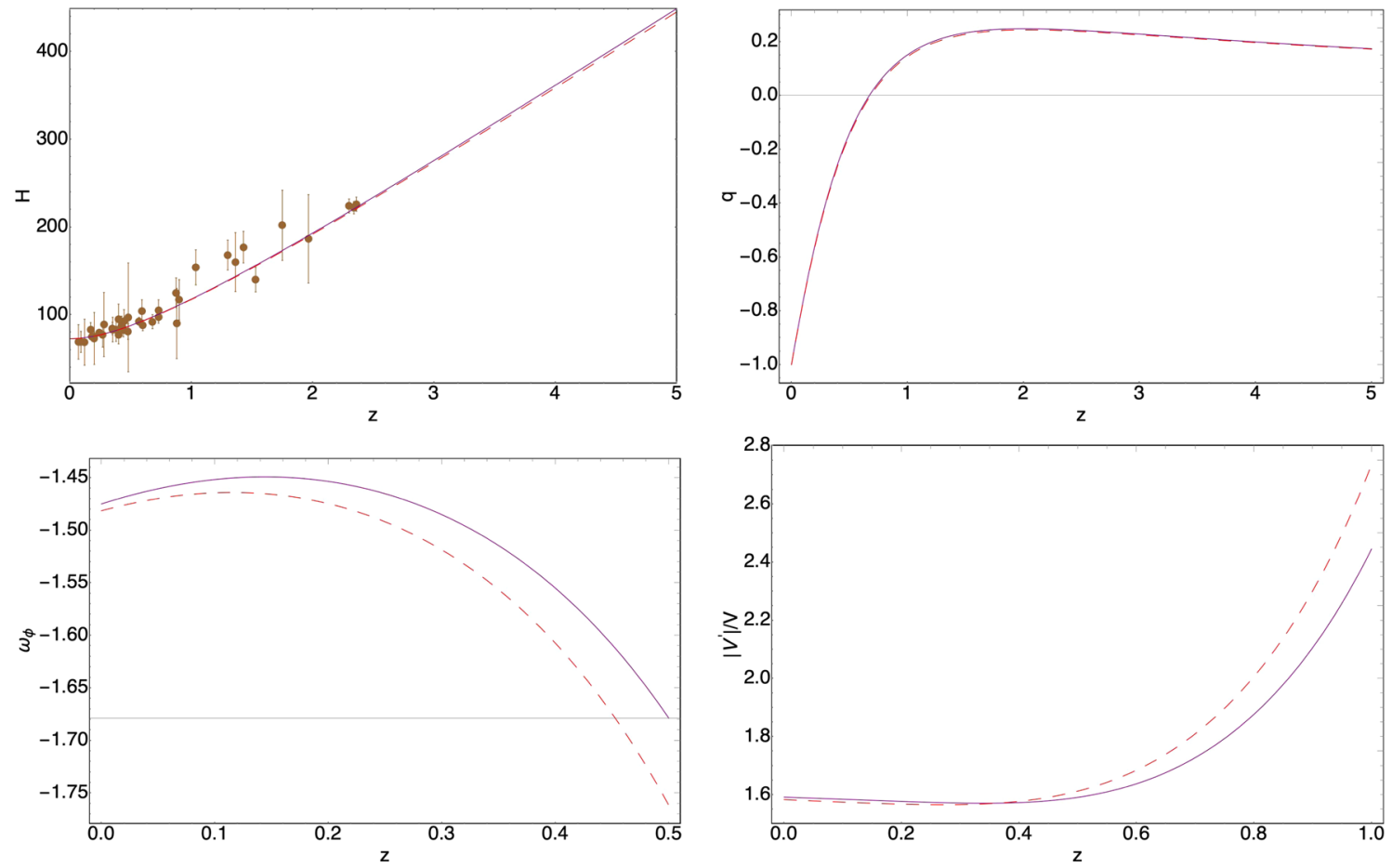

Fig. 2 The graphic behavior of the Hubble parameter in comparison with known $H(z)$ data given in Table 1 is presented on the left hand side plot of the top panel. The plot on the right hand side corresponds to the behavior of the deceleration parameter. The left hand side plot of the bottom panel depicts the behavior of the $\omega_{\phi}=P_{\phi} / \rho_{\phi}$ equation of state parameter describing the scalar field dark energy model. The right hand side plot of the bottom panel shows the graphical behavior of SC2 Eq. (2). In all cases the purple curve is a plot for the best fit values of the model parameters, when $z \in[0,2.5]$, while the dashed red curve

has been chosen for the case when $z \in[0,5]$. The red dots represent the known observational $H(z)$ data (30 samples deduced from the differential age method and 10 samples obtained from the radial BAO method), the same as in Ref. [30]. The redshift range for $\omega_{\phi}$ has been chosen in order to make it possible to compare our results with those presented in [30]. The Bayesian Machine Learning procedure has been devised for the model given by Eq. (14). The best fit values used in the analysis can be found in Table 2

Table 2 Best fit values and $1 \sigma$ errors estimated for Model 1, Eq. (14), for $z \in[0,2.5]$ and for $z \in[0,5]$, respectively. The results depicted in the first part of the table are obtained from the generative process based on SC1, Eq. (1). The second part of the table corresponds to the results of the fit when the theoretical form of the expansion rate, Eq. (14), is used

\begin{tabular}{llll}
\hline$H(z)=H_{0}+H_{1} \frac{z^{2}}{1+z}$ & $\Omega_{d m}$ & $H_{0}$ & $H_{1}$ \\
\hline When $z \in[0,2.5]$ & $0.322 \pm 0.01$ & $72.59 \pm 1.21 \mathrm{~km} / \mathrm{s} / \mathrm{Mpc}$ & $90.34 \pm 1.61 \mathrm{~km} / \mathrm{s} / \mathrm{Mpc}$ \\
When $z \in[0,5]$ & $0.325 \pm 0.012$ & $72.55 \pm 1.06 \mathrm{~km} / \mathrm{s} / \mathrm{Mpc}$ & $89.43 \pm 1.8 \mathrm{~km} / \mathrm{s} / \mathrm{Mpc}$ \\
\hline$H(z)=H_{0}+H_{1} \frac{z^{2}}{1+z}$ & $\Omega_{d m}$ & $H_{0}$ & $H_{1}$ \\
\hline When $z \in[0,2.5]$ & - & $71.524 \pm 0.5 \mathrm{~km} / \mathrm{s} / \mathrm{Mpc}$ & $90.654 \pm 0.5 \mathrm{~km} / \mathrm{s} / \mathrm{Mpc}$ \\
When $z \in[0,5]$ & - & $71.094 \pm 0.5 \mathrm{~km} / \mathrm{s} / \mathrm{Mpc}$ & $91.83 \pm 0.1 \mathrm{~km} / \mathrm{s} / \mathrm{Mpc}$ \\
\hline
\end{tabular}

Specifically, first of all, we have proven that, incorporating both Bayesian (Probabilistic) Machine Learning and string Swampland criteria, we are definitely able to constrain cosmological models. Then, we have validated the fit results using observational expansion rate data already available. Moreover, our procedure allowed to demonstrate that the string Swampland criteria is in a certain tension with recent observations and the understanding of dark energy, in the scheme of GR with standard matter and a dark energy splitting approach. Moreover, we noticed a spontaneous sign switch in the dark energy equation of state parameter, when the scalar field net excursion leaves the domain $z \in[0,2.5]$.

On the other hand, we should note that our results come from a specific form of the expansion rate, constructed in the 


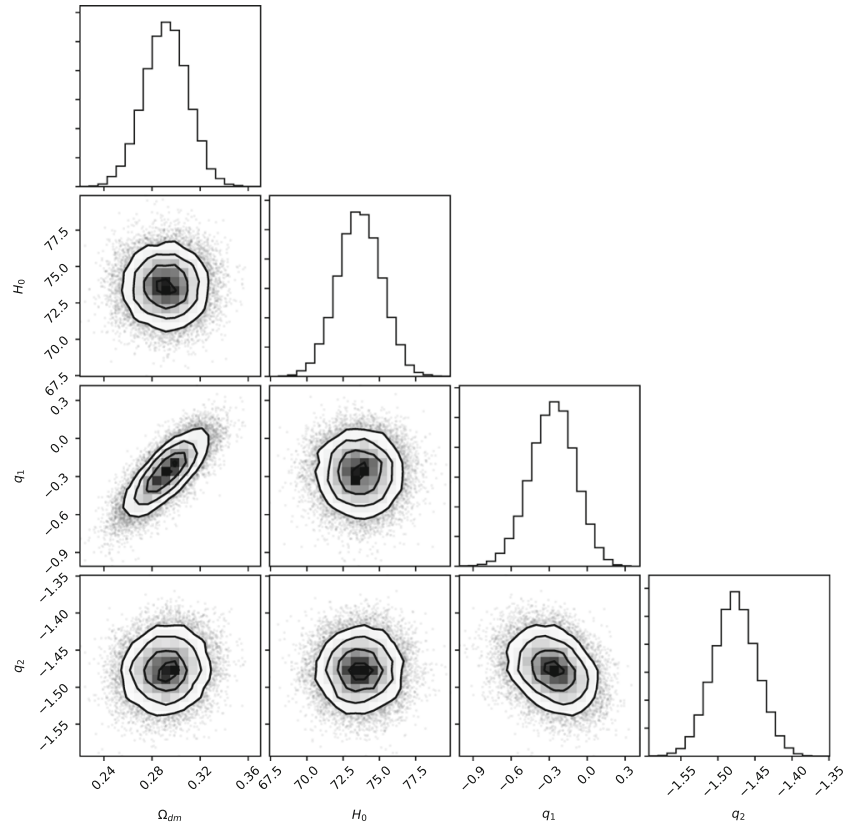

Fig. 3 The contour map of the model given by Eq. (16), for $z \in[0,2.5]$, is given by the left hand side plot. The one on right hand side corresponds to the contour map of the same model, for $z \in[0,5]$. The best fit values of the model parameters are, respectively, $\Omega_{d m}=0.322 \pm 0.01$, $H_{0}=73.64 \pm 1.5 \mathrm{~km} / \mathrm{s} / \mathrm{Mpc}, q_{1}=-0.27_{-0.172}^{+0.181}, q_{1}=-1.48 \pm 0.03$, for $z \in[0,2.5]$, while for $z \in[0,5]$ they are found to be $\Omega_{d m}=$

Bayesian (Probabilistic) Machine Learning approach. It must be stressed once more that, in the generative based Bayesian (Probabilistic) Machine Learning procedure, we generated the field traverse and used it as the "dataset" to constrain the model. In this regard, it would be interesting to investigate other expansion rate models, to reveal whether and how the specific approach being used will affect the Swampland related results. Aiming at this goal, we consider in addition an alternative toy model, in this case, a model parametrizing the deceleration parameter. Results for this case are described in the next section.

\subsection{Model with a given $q(z)$ parameterization}

The second toy model we consider relies on a deceleration parameter parameterization, given by the following expression [70]

$q(z)=\frac{1}{2}+\frac{q_{1} z+q_{2}}{(z+1)^{2}}$,

where $q_{1}$ and $q_{2}$ are the free parameters to be determined. It is easy to see, that the expansion rate of the Universe with such a deceleration parameter has the form

$H(z)=H_{0}(z+1)^{3 / 2} e^{\frac{z\left(q_{1} z+q_{2}(z+2)\right)}{2(z+1)^{2}}}$,

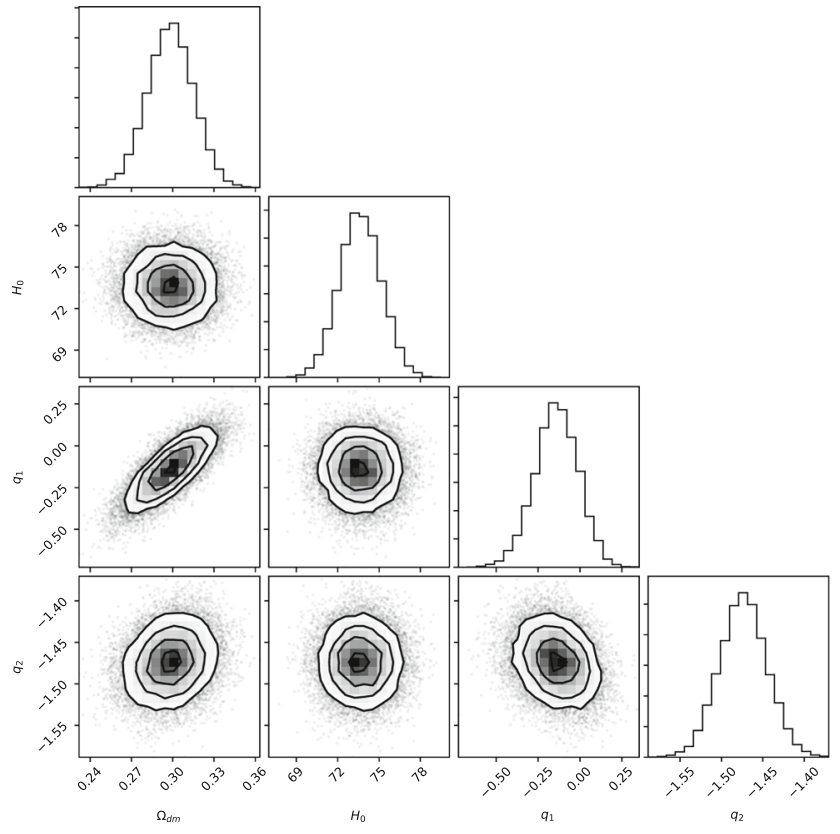

$0.298 \pm 0.0167, H_{0}=73.63_{-1.52}^{+1.476} \mathrm{~km} / \mathrm{s} / \mathrm{Mpc}$ and $q_{1}=-0.139_{-0.127}^{+0.13}$ and $q_{2}=-1.47 \pm 0.029$. The generative process has been constructed using SC1, Eq. (1), where $H(z)$ is given by Eq. (16). It has been used to get $H^{\prime}$ and $H^{\prime \prime}$. We refer to the PyMC3 documentation for more details on how the marginalization and the error estimations can be achieved during the learning process

where $H_{0}, q_{1}$ and $q_{2}$ are the parameters of the model, this one having one parameter more, as compared to the previous one. Following the same procedure as in case of the first model, we have been able to constrain the new parameters. We have found that

- When we consider $z \in[0,2.5]$, then the best fit values and $1 \sigma$ errors are $\Omega_{d m}=0.322 \pm 0.01, H_{0}=73.64 \pm 1.5$ $\mathrm{km} / \mathrm{s} / \mathrm{Mpc}, q_{1}=-0.27_{-0.172}^{+0.181} q_{1}=-1.48 \pm 0.03$.

- When we consider $z \in[0,5]$, then the best fit values and $1 \sigma$ errors are $\Omega_{d m}=0.298 \pm 0.0167, H_{0}=73.63_{-1.52}^{+1.476}$ $\mathrm{km} / \mathrm{s} / \mathrm{Mpc}$ and $q_{1}=-0.139_{-0.127}^{+0.13}$ and $q_{2}=-1.47 \pm$ 0.029 .

The contour maps of the model given by Eq. (16), for $z \in[0,2.5]$ and $z \in[0,5]$, can be found in Fig. (3). Similar to the previous case, our results prove that the string Swampland criteria, Eqs. (1) and (2), is in tension with GR with standard dark matter and quintessence dark energy splitting. On the other hand, similarly to the previous case, we get the hint that it could be possible to build an effective theory not ending up in the Swampland, but then the dark energy in the lowredshift Universe should have phantom nature. Moreover, the behavior of the $\omega_{\phi}=P_{\phi} / \rho_{\phi}$ equation of state parameter, describing the scalar field dark energy model, would be in 

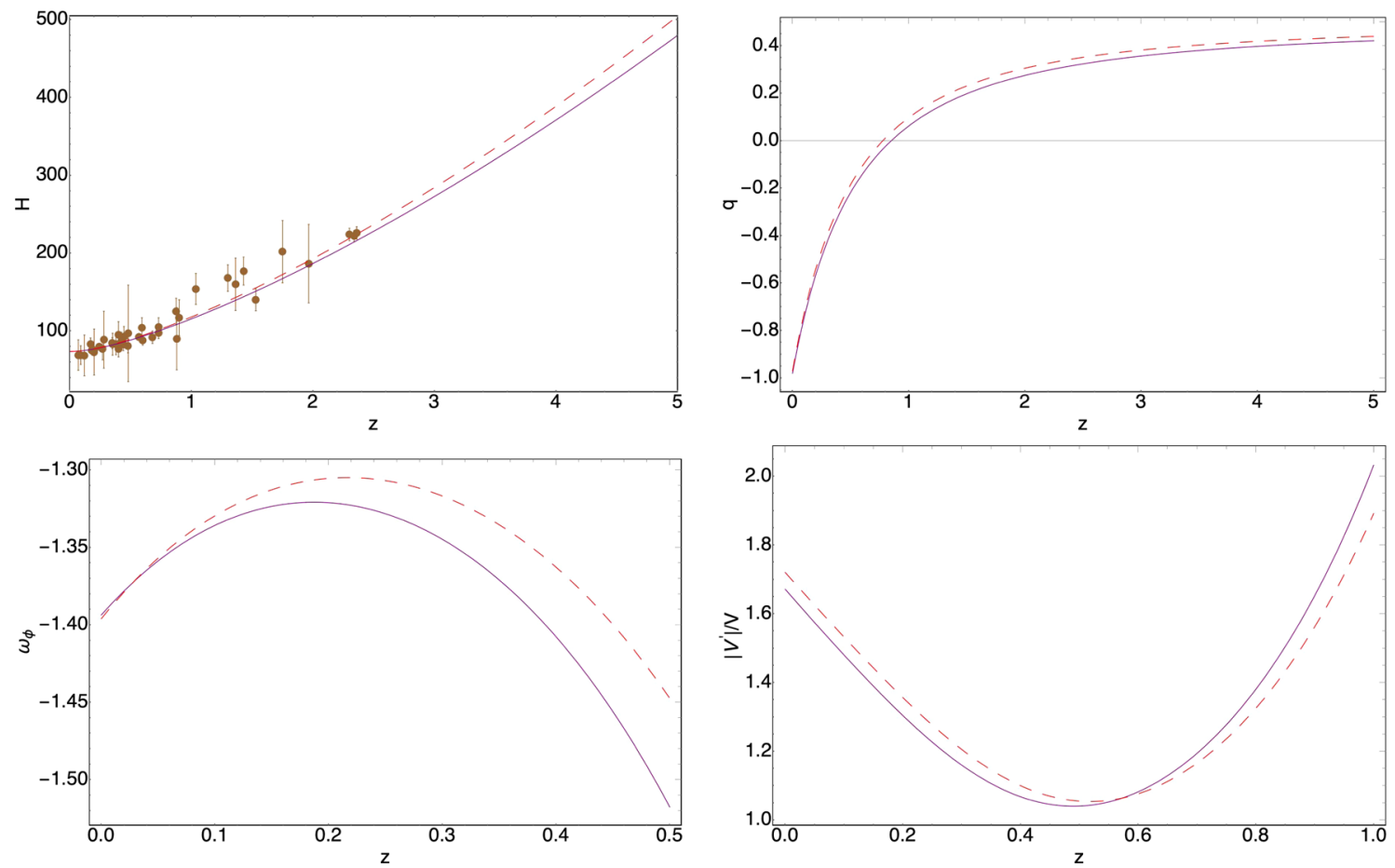

Fig. 4 A graphic behavior of the Hubble parameter, compared with known $H(z)$ data presented in Table 1, is depicted on the left hand side plot of the top panel. The behavior of the deceleration parameter is represented on the right hand side. The left hand side plot of the bottom panel corresponds to the behavior of the $\omega_{\phi}=P_{\phi} / \rho_{\phi}$ equation of state parameter describing the scalar field dark energy model. Further, the right hand side plot of the bottom panel shows the graphic behavior of SC2, Eq. (2). In all cases, the purple curve is the plot for the best fit values of the parameters of the model, when $z \in[0,2.5]$; while the dashed

red curve corresponds to the case when $z \in[0,5]$. The red dots represent the known observational $H(z)$ data $(30$ samples deduced from the differential age method and ten samples obtained from the radial BAO method), and it is the same as in [30]. The redshift range for $\omega_{\phi}$ has been chosen in order to make it possible to compare our results with those given in [30]. The Bayesian Machine Learning procedure has been constructed for the model given by Eq. (16). The best fit values used in the analysis are to be found in Table 3

very good agreement with the results of [30], too. Moreover, during the validating of our fit results with the expansion rate data, we have found that, most likely, a high-redshift tension between the expansion rate, Eq. (16), based on theoretical results and observations, will arise. This has been observed when the field traverse has been limited to $z \in[0,2.5]$. On the other hand, when the field traverse extends to $z \in[0,5]$, the mentioned tension actually disappears. In other words, the model can explain the accelerated expansion of the low redshift Universe and the transition to that phase. Moreover, it is free from the $H_{0}$ tension problem due to the best fit values of the model parameters, and can explain the BOSS result for the Hubble parameter at $z=2.34$.

All these results can be seen in Fig. (4), where a comparison of the ones corresponding to $z \in[0,2.5]$, with those coming from $z \in[0,5]$, limiting the field traverse, can be found, too. In addition, the results summarizing how the two redshift ranges considered for the field traverse will affect the model parameters constraints, can be found in the first part of Table 3. The second part of the table shows the corresponding constrains when the generative process is based

on Eq, (16). This is another way of showing that the string Swampland criteria for a lower redshift dark energy dominated Universe is most likely in tension with the up to now available observations.

To finish this section, we should stress again, that an interplay between Bayesian (Probabilistic) Machine Learning and the Swampland criteria can provide a working pipeline allowing to constrain the free parameters of the underlying theory. Moreover, it can identify the position of the theory in phase space, clearly showing if it belongs (or not) to the Swampland and, moreover, if it will, or not, end up there. The two toy models discussed yield already clear enough results, which demonstrate how our approach can be used successfully, to extract valuable information using minimal information. Certainly, several other questions still arise, which should be clarified before a final conclusion can be reached, but it seems already clear that we have started to explore a very promising direction of study, which reveals a connection between Swampland and cosmology. Further results on this topic will be reported in forthcoming papers. Finally, similar to the previous model, we observe again a spontaneous sign 
Table 3 Best fit values and $1 \sigma$ errors estimated for Model 2, Eq. (16), for $z \in[0,2.5]$ and for $z \in[0,5]$, respectively. The results presented in the first part of the table are obtained from the generative process based on SC1, Eq. (1). The second part of the table represents the fit results when the theoretical form of the expansion rate, Eq. (16), is used in the generative process. In our analysis we used $\Omega_{d m} \in[0.2,0.5]$, $H_{0} \in[65.0,80.0], q_{1} \in[-1.0,1.0]$ and $q_{2} \in[-3.0,3.0]$ flat priors. We refer to the PyMC3 documentation for more details on how the marginalization and the error estimations can be achieved during the learning process

\begin{tabular}{lllll}
\hline$q(z)=\frac{1}{2}+\frac{q_{1} z+q_{2}}{(z+1)^{2}}$ & $\Omega_{d m}$ & $H_{0}$ & $q_{1}$ & $q_{2}$ \\
\hline When $z \in[0,2.5]$ & $0.292 \pm 0.0181$ & $73.64 \pm 1.5 \mathrm{~km} / \mathrm{s} / \mathrm{Mpc}$ & $-0.27_{-0.172}^{+0.181}$ & $-1.48 \pm 0.03$ \\
When $z \in[0,5]$ & $0.298 \pm 0.0167$ & $73.63_{-1.52}^{+1.476} \mathrm{~km} / \mathrm{s} / \mathrm{Mpc}$ & $-0.139_{-0.127}^{+0.13}$ & $-1.47 \pm 0.029$ \\
\hline$q(z)=\frac{1}{2}+\frac{q_{1} z+q_{2}}{(z+1)^{2}}$ & $\Omega_{d m}$ & $H_{0}$ & $q_{1}$ & \\
\hline When $z \in[0,2.5]$ & - & $73.23 \pm 0.23 \mathrm{~km} / \mathrm{s} / \mathrm{Mpc}$ & $0.043 \pm 0.056$ & $-1.48 \pm 0.026$ \\
When $z \in[0,5]$ & - & $73.56 \pm 0.05 \mathrm{~km} / \mathrm{s} / \mathrm{Mpc}$ & $-0.141 \pm 0.0078$ & $-1.49 \pm 0.0054$ \\
\hline
\end{tabular}

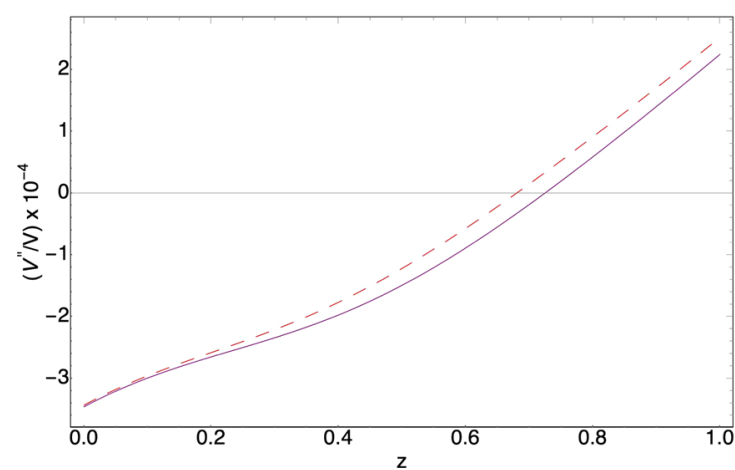

Fig. 5 A graphical behavior of the SC2, Eq. (3), for the first model given Eq. (14) is depicted on the left hand side plot. On the right hand side plot, the behavior of the SC2, Eq. (3), for the second model given Eq. (16) is represented. In all cases, the purple curve is the plot for

switch in the dark energy equation of state parameter. This is an interesting phenomenon requiring further analysis, too. Eventually, our future analysis indicates that the SC2 given by Eqs. (2) and (3) carries qualitatively identical information about our low-redshift dark energy dominated Universe. The graphic behavior of the SC2 given by Eq. (3) can be found in Fig. (5).

\section{Conclusions}

We say nothing new when stating that Machine Learning, and in general Artificial Intelligence, has brought new insights in data analysis and data engineering, computer, and vision science. Nowadays, thanks to the very robust algorithms developed, machine learning allows our computers to interact with us, talk to us, and we even build self-driving cars. Definitely, in the near future, we will witness more serious achievements based on these algorithms, which will allow us to explore new research fields and tasks. The study in this paper belongs to a series of works, where machine learning is being used in the study of hard cosmological and astrophysical prob-

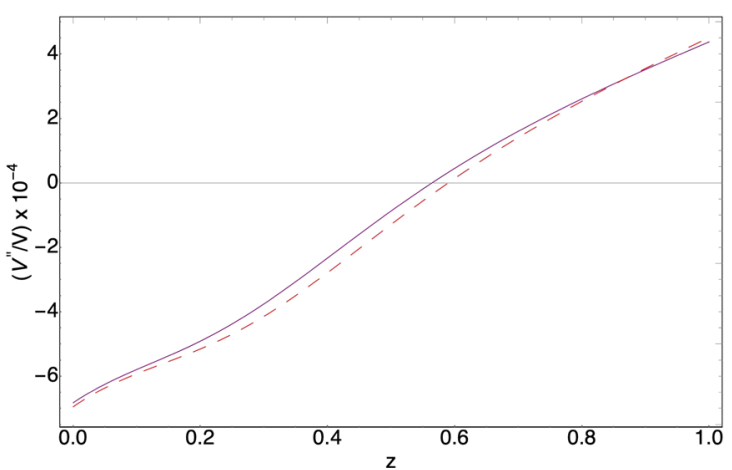

the best fit values of the parameters of the models, when $z \in[0,2.5]$; while the dashed red curve corresponds to the case when $z \in[0,5]$. The best fit values used in the analysis are to be found in Tables 2 and 3 , respectively

lems, very difficult to solve otherwise. The approach we used here is, however, quite different from the ones employed in other works, where observational data have been used to train Machine Learning algorithms. Our approach is a Bayesian (Probabilistic) Machine Learning process, which allows to discriminate between theories by using modelbased forward simulations. In this way, we incorporate our prior believe directly within the posterior distribution. Then, during the Bayesian (Probabilistic) Learning process, the priors are updated through mock data generation and the Machine Learning algorithm. Recently, it has been successfully applied to study the $H_{0}$ tension problem, by using single viscous fluid models, and also to constrain the cosmic opacity of the Universe at different redshift ranges [47,48]. Having realized the power of the approach through these previous successes, in this paper we have taken a step forward, by incorporating into the analysis string Swampland criteria. More specifically, starting from these criteria and applying then Bayesian (Probabilistic) Machine Learning, we have been able to constrain the underlying effective theory and to determine if the resulting best theory for describing low 
redshift behavior of a dark energy dominated Universe will end up in the Swampland or not.

Our work was motivated by the results in [30], where a Gaussian Process had been used to study string Swampland criteria in a model-independent way, directly using observational data without involving a specific dark energy model and the potential describing the scalar field. One of the results from that reconstruction was to realize that an effective theory being in the Swampland could end up or not there. Also, it could start not being in the Swampland but end up either inside or outside of it. However, that mentioned study was totally limited to the redshift range were data are already available. On the contrary, using, as we do here, Bayesian (Probabilistic) Machine Learning, we can overcome these limitations. On the other hand, since the starting point for us is [30], we needed one more step in order to perform the generative process based on Bayesian (Probabilistic) Machine Learning. Specifically, one needs the form of the expansion rate to complete Eqs. (1) and (2) (or Eq. (3)) describing the string Swampland criteria, since they can be expressed in terms of $H(z), H^{\prime}(z)$ and $H^{\prime \prime}(z)$. Indeed, the consideration of two different models describing $H(z)$ has allowed us to constrain the background dynamics for each of them, and to determine whether the models are in the Swampland or not. It should be mentioned that the results we have obtained are fully consistent with [30], what indicates that the string Swampland criterion is in tension with GR with standard dark matter and quintessence dark energy splitting. Another conclusion is that it should be possible to build an effective theory with phantom dark energy well consistent with the string Swampland criteria. As, moreover, astronomical observations continue supporting the quintessence nature of dark energy, this could be also seen as an indication that GR with standard dark matter and quintessence dark energy splitting should eventually end up in the Swampland. Applying Bayesian Machine Learning for two toy models we obtained results in good agreement with the results of the model-independent analysis [30]. Therefore, combining two results allows us to become more confident that the string Swampland criteria might be in tension with recent observations indicating that phantom dark energy cannot be in the Swampland. On the other hand, it indicates that the true nature of both models have been explored fully.

Let us summarize from where we started and what we have achieved up to now. First of all, we have demonstrated that incorporating Bayesian (Probabilistic) Machine Learning and string Swampland criteria, we can constrain cosmological models. Then, we have validated the results of the fit by using available expansion rate data. Further, we have seen that the string Swampland criteria and GR with standard dark matter and quintessence dark energy splitting are in tension. We observed also that, most likely, the theory will not end up in the Swampland, provided we introduce phantom dark energy. All this can be a hint that a simple splitting of the energy source between standard matter and dark energy used in the studies is not applicable on cosmological scales. On the other hand, the results come from a specific form of the expansion rate, Eq. (14), constructed with the Bayesian (Probabilistic) Machine Learning approach. In this regard, it becomes interesting to investigate how the form of the expansion rate introduced in the Bayesian (Probabilistic) Machine Learning approach will affect the Swampland related results. To this end we have studied a second model, where a parameterization of the deceleration parameter has been used, yielding another parameterization of the expansion rate, Eq. (16). The conclusion is the same, but with one difference: that the model with Eq. (16) is preferable since it can solve the $H_{0}$ tension problem and explain the BOSS result for the $H(z)$ at $z=2.34$.

In our analysis, the two redshift ranges $z \in[0,2.5]$ and $z \in[0,5]$ have been considered. Since our results have been validated and compared with the available expansion rate data, we forecast that, most likely, high redshift observations of the expansion rate will not affect our conclusions on the string Swampland criteria. Moreover, we have used again the Bayesian (Probabilistic) Machine Learning approach, with a $H(z)$ generative process, to constrain the parameters $H_{0}$ and $H_{1}$ for the first model, Eq. (14), and $H_{0}, q_{1}$ and $q_{2}$ for the second one, Eq. (16). The results indicate that the same approach imposes very tight constraints on these parameters, as compared with the constraints obtained using SC1, Eq. (1). This is another evidence of the mentioned existing tension and could be a starting point to finally understanding why it is there and to finding a way to solve it. Moreover, we have found that the SC2 given by Eq. (2) and Eq. (3) carries qualitatively identical information about our low-redshift dark energy dominated Universe.

To end up, we remark that an interplay between Bayesian (Probabilistic) Machine Learning and the Swampland criteria can provide a working pipeline allowing to constrain the free parameters of the underlying theory. It can identify the position of the theory on phase space, indicating whether it is in the Swampland or not, and also whether eventually it will end up, or not, there. Using two simple toy models, we have already proven that this approach can be actually useful in this respect, and that it can be used to extract valuable information from a minimal input information. Indeed, there are several additional questions that should be clarified before we can get a final assessment of the power of the method; but it seems clear that we have initiated a promising path, which has already revealed novel connections between the Swampland and cosmology. We expect to report more results on this topic in forthcoming papers, with particular attention on the construction of a specific form of $H(z)$ exhibiting Swampland-NotSwampland 
and NotSwampland-Swampland transitions, as they are not in principle forbidden, according to the study of [30].

The last point to be mentioned and which will require also future study is the spontaneous sign switch in the dark energy equation of state parameter, which we have observed when the field traverses are in the $z \in[0,5]$ redshift range. We can have several reasons to see the spontaneous sign switch in the dark energy equation of state parameter. The understanding of it is very important. First of all, it is important to understand whether or not this is a machine learning-related issue allowing to develop a correct physical intuition. We do hope that very soon with a new paper we can address this question very thoroughly.

Finally, it should be mentioned again, that in this study we only used the field traverse as a "dataset" simulated by the Bayesian (Probabilistic) Machine Learning allowing to incorporate the Swampland criteria and underlying theory. In this regard, it is clear how and why the suggested new approach is different from previous studies, indicating that we can explore more dark parts of the Swampland, which previously was not possible at all to do. One of them would be the extended search for a solution of the $H_{0}$ tension problem in terms of different $H(z)$ parameterizations, as it has been done here.

Acknowledgements This work has been partially supported by MINECO (Spain), project FIS2016-76363-P, and by AGAUR (Catalan Government), project 2017-SGR-247. The authors would like to thank the referee for valuable comments which helped to improve the manuscript. We also thank Eoin Colgain for interesting comments.

Data Availability Statement This manuscript has no associated data or the data will not be deposited. [Authors' comment: In this paper, only mock data generated from the discussed theoretical models have been. A detailed description of how the mock data can be generated also has been presented. Therefore, there is no reason to deposit any mock data which can be easily generated.]

Open Access This article is licensed under a Creative Commons Attribution 4.0 International License, which permits use, sharing, adaptation, distribution and reproduction in any medium or format, as long as you give appropriate credit to the original author(s) and the source, provide a link to the Creative Commons licence, and indicate if changes were made. The images or other third party material in this article are included in the article's Creative Commons licence, unless indicated otherwise in a credit line to the material. If material is not included in the article's Creative Commons licence and your intended use is not permitted by statutory regulation or exceeds the permitted use, you will need to obtain permission directly from the copyright holder. To view a copy of this licence, visit http://creativecomm ons.org/licenses/by/4.0/.

Funded by SCOAP ${ }^{3}$.

\section{References}

1. S. Nojiri, S.D. Odintsov, V.K. Oikonomou, Phys. Rept. 692, 1 (2017)
2. S. Nojiri, S.D. Odintsov, eConf C 0602061, 06 (2006) [Int. J. Geom. Meth. Mod. Phys.4, 115 (2007)]

3. S. Nojiri, S.D. Odintsov, Phys. Rept. 505, 59 (2011)

4. E. Elizalde, S. Nojiri, S.D. Odintsov, D. Saez-Gomez, Eur. Phys. J. C 70(351), 351 (2010)

5. E. Elizalde, R. Myrzakulov, V.V. Obukhov, D. Saez-Gomez, Class. Quantum Gravit. 27, 095007 (2010)

6. G. Cognola, E. Elizalde, S. Nojiri, S. D. Odintsov, L. Sebastiani, S. Zerbini., Phys. Rev. D 77, 046009 (2008)

7. S. Kachru, R. Kallosh, A.D. Linde, S.P. Trivedi, Phys. Rev. D 68, 046005 (2003)

8. V. Balasubramanian, P. Berglund, J.P. Conlon, F. Quevedo, JHEP 03, 007 (2005)

9. A. Westphal, JHEP 03, 102 (2007)

10. X. Dong, B. Horn, E. Silverstein, G. Torroba, Class. Quantum Gravit. 27, 245020 (2010)

11. M. Rummel, A. Westphal, JHEP 01, 020 (2012)

12. J. Blbck, U. Danielsson, G. Dibitetto, JCAP 1403, 003 (2014)

13. M. Cicoli et al., JHEP 05, 001 (2014)

14. J.M. Maldacena, C. Nunez, Int. J. Mod. Phys. A 16, 822 (2001)

15. P. K. Townsend, (2020). arXiv:hep-th/0308149

16. M.P. Hertzberg et al., JHEP 12, 095 (2007)

17. C. Caviezel, T. Wrase, M. Zagermann, JHEP 04, 011 (2010)

18. B. de Carlos, A. Guarino, J.M. Moreno, JHEP 01, 012 (2010)

19. G. Shiu, Y. Sumitomo, JHEP 09, 052 (2011)

20. S.R. Green et al., Class. Quantum Gravit. 29, 075006 (2012)

21. F.F. Gautason, D. Junghans, M. Zagermann, JHEP 06, 029 (2012)

22. D. Kutasov et al., Phys. Rev. Lett. 115, 071305 (2015)

23. D. Junghans, JHEP 06, 132 (2016)

24. D. Andriot, J. Blbck, JHEP 03, 102 (2017)

25. J. Moritz, A. Retolaza, A. Westphal, Phys. Rev. D 97, 046010 (2018)

26. U.H. Danielsson, T. Van Riet, (2020). arXiv:1804.01120

27. H. Ooguri, C. Vafa, Nucl. Phys. B 766, 21 (2007)

28. G. Obied et al., (2020). arXiv:1806.08362

29. H. Ooguri, E. Palti, G. Shiu, C. Vafa, Phys. Lett. B 788, 180 (2019)

30. E. Elizalde, M. Khurshudyan, Phys. Rev. D 99, 103533 (2019)

31. G.S. Sharov, E.S. Sinyakov, (2020). arXiv:2002.03599

32. H. Amirhashchi, A.K. Yadav, (2020). arXiv:2001.03775

33. M. Braglia et al., (2020). arXiv:2004.1116

34. W.L. Kimmy Wu et al., (2020). arXiv: 2004.10207

35. G. Alestas et al., (2020). arXiv:2004.08363

36. D. Wang, D. Mota, arXiv:2003.10095

37. N. Blinov et al., (2020). arXiv: 2004.06114

38. E.K. Li et al., (2020). arXiv:1911.12076

39. J. Sakstein, M. Trodden, Phys. Rev. Lett. 124, 161301 (2020)

40. M.H.P.M. van Putten, (2020). arXiv:1707.02588

41. E. Di Valentino et al., Phys. Rev. D 101, 063502 (2020)

42. E. Di Valentino et al., (2020). arXiv:1908.04281

43. E. Di Valentino et al., (2020). arXiv:2005.12587

44. Y.F. Cai et al., Astrophys. J. 888, 62 (2020)

45. R.C. Nunes, JCAP $\mathbf{0 5}, 052$ (2018)

46. E. Elizalde et al., (2020). arXiv:2104.01077

47. E. Elizalde et al., Phys. Rev. D 102(12), 123501 (2020)

48. E. Elizalde, M. Khurshudyan, (2020). arXiv:2006.12913

49. T. Yang, Phys. Rev. D 102, 083511 (2020)

50. B. Bayarsaikhan et al., (2020). arXiv:2005.11171

51. S.D. Odintsov, V.K. Oikonomou, Phys. Lett. B 805, 135437 (2020)

52. L.A. Anchordoqui et al., Phys. Rev. D 101, 083532 (2020)

53. P. Agrawal et al., (2020). arXiv:1906.08261

54. V. Aragam et al., JCAP 04, 022 (2020)

55. S. Brahma, Md. Wali Hossain, JHEP 06, 070 (2019)

56. J.J. Heckman et al., Fortsch. Phys. 67, 11 (2019)

57. A. Banerjee et al., (2020). arXiv:2006.00244

58. E.O. Colgan, H. Yavartanoo, (2020). arXiv:1905.02555

59. E.O. Colgain et al., Phys. Lett. B 793, 126-129 (2019) 
60. S.K. Garg, C. Krishnan, J. High Energy Phys. 2019, 75 (2019)

61. L. Heisenberg et al., PRD 98, 123502 (2018)

62. Y. Akrami et al., Fortsch. Phys. 67, 1800075 (2019)

63. S.D. Odintsov, V.K. Oikonomou, EPL 126(2), 20002 (2019)

64. R. Arjona, S. Nesseris, (2020). arXiv:2012.12202

65. A. Barrau et al., (2020). arXiv:2101.02942

66. L. Heisenberg et al., (2020). arXiv:2003.13283

67. R.-G. Cai et al., Phys. Dark Univ. 26, 100387 (2019)

68. M.-J. Zhang, J.-Q. Xia, JCAP 1612(12), 005 (2016)
69. J. Salvatier et al., (2020) PeerJ Comput. Sci. 2, e55. https://doi.org/ 10.7717/peerj-cs.55

70. Y. Gong, A. Wang, Phys. Rev. D 73, 083506 (2006) 